\title{
Pancreatic neuroendocrine tumours: Established role of endoscopic ultrasound in high-risk populations
}

\author{
Eric C Lam MD FRCPC
}

$\mathrm{P}$ ancreatic neuroendocrine tumours (PNETs) are rare tumours of the pancreas that arise from cells that produce hormonally active substances. Insulin and gastrin are the most common hormone-producing tumours that are associated with symptoms. A proportion of these tumours can develop without symptoms and are incidentally found at the time of abdominal imaging, or only become symptomatic when the tumour is large.

Certain genetic syndromes are associated with a high prevalence of PNETs. Multiple endocrine neoplasm type 1 (MEN1) is the most common genetic syndrome associated with PNETs. The tumours can be multifocal, ranging in size and function. Often, MEN1 patients have a high prevalence of other tumours, such as adrenal and pituitary tumours.

In this issue of The Canadian Journal of Gastroenterology, Alsohaibani et al (pages 817-820) report a retrospective study of 14 PNET patients who underwent preoperative assessment with endoscopic ultrasound (EUS). They also discuss the impact that EUS had on surgical decision-making. The initial workup for nearly all patients consisted of computed tomography (CT) scanning and EUS, while others had other preoperative workups, such as magnetic resonance imaging and octreotide scintigraphy. EUS detected PNETs in all 14 cases $(100 \%)$, whereas the CT detection rate was $77 \%$. Furthermore, among those with multifocal PNETs, the extent of pancreatic involvement was underestimated by CT imaging in three cases. As a result, the decision to undergo surgery was changed in $36 \%$ of patients and the extent of surgery required was changed in $50 \%$ of patients.

There are drawbacks to the study by Alsohaibani et al. No inferences can be made about the efficacy of EUS in impacting the role of surgery because of the small numbers of patients and the retrospective, descriptive study design. However, because PNETs are rare (estimates of one per 100,000 population), to accumulate more cases would require years, during which time imaging technology and surgical techniques would most certainly change. A multicentre review would be the best way to investigate whether EUS truly impacts surgery.

Although the authors state that there are drawbacks to their retrospective case series, the results speak to the detection limits of existing imaging techniques. Many PNETs are vascular lesions and often enhance to the same degree as adjacent pancreatic tissue, thereby evading detection by contrast CT. Although all PNETs have somatostatin receptors, the elusive tumours are small and there is variable binding of somatostatin in them. Therefore, current indium-based scintigraphy is unable to detect the small lesions with variable somatostatin binding due to a lack of resolution. More promising data are emerging on radiopharmaceuticals with variable affinity somatostatin receptor analogues (1). Finally, magnetic resonance imaging has a better sensitivity than CT scanning, but small lesions can also be missed by this modality. Only EUS, which has an axial resolution of $0.4 \mathrm{~mm}$ at $7.5 \mathrm{MHz}$, can reliably detect these small lesions and provide a cytological diagnosis by fine needle aspiration (2).

Is EUS an essential modality in all PNETs, or all pancreatic masses, for that matter? Risk-stratifying patients would determine which patients would benefit the most from EUS. Patients with MEN1 are distinctly different from those who present with no such history. Indeed, in the study by Alsohaibani et al, all patients who were found to have multifocal disease had MEN1. Some authorities have suggested that asymptomatic MEN1 patients should be screened to detect multifocal disease (3). Because not all MEN1-related PNETs are functional tumours, detection before they become symptomatic is key.

As for the PNETs not associated with MEN1 or other genetic syndromes, this group is usually detected as an incidental finding on cross-sectional imaging. Unless these tumours undergo cystic degeneration or cause compressive biliary symptoms, they are essentially asymptomatic. There is an increasing trend of detecting nonfunctional tumours. This group of patients will become more important in the future (4). With the current state of cross-sectional imaging, a high index of suspicion is required to make this diagnosis with EUS providing the cytological confirmation by fine needle aspiration.

There is a well-established role for EUS in pancreatic masses. Cross-sectional imaging will still be the test of first contact and is needed to assess distant metastatic disease. However, the article by Alsohaibani et al has reiterated the essential role of EUS in providing a more detailed evaluation of pancreatic masses.

\section{REFERENCES}

1. Gotthardt M, Boermann OC, Behr TM, et al. Development and clinical application of peptide-based radiopharmaceuticals. Curr Pharm Des 2004;10:2951-63.

2. Hwang JH, Kimmey MB. Principles of ultrasound. In: Hawes RH, Fockens P, eds. Endosonography. Philadelphia: Saunders Elsevier, 2006:3-16.

3. Thomas-Marquez L, Murat A, Delemer B, et al. Prospective endoscopic ultrasonographic evaluation of the frequency of nonfunctioning pancreaticoduodenal endocrine tumors in patients with multiple endocrine neoplasia type 1 . Am J Gastroenterol 2006;101:266-73.

4. Jani N, Khalid A, Kaushik N, et al. EUS-guided FNA diagnosis of pancreatic endocrine tumors: New trends identified. Gastrointest Endosc 2008;67:44-50.

Department of Gastroenterology, University of British Columbia, Vancouver, British Columbia

Correspondence: Dr Eric C Lam, Pacific Gastroenterology Associates, 770 - 1190 Hornby Street, Vancouver, British Columbia V6J 5 M2.

Telephone 604-688-6332 ext 224, fax 604-689-2004, e-mail ecslam@shaw.ca

Received for publication July 16, 2008. Accepted July 21, 2008 


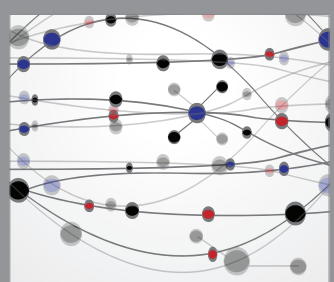

The Scientific World Journal
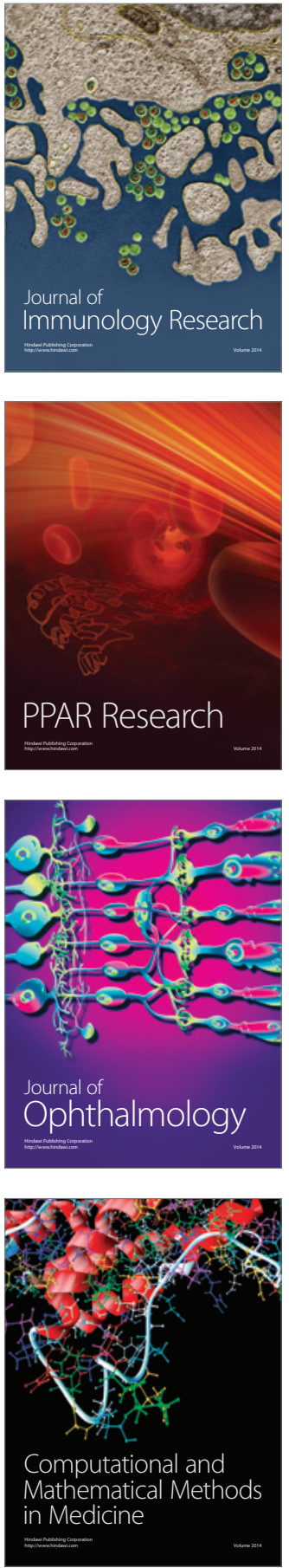

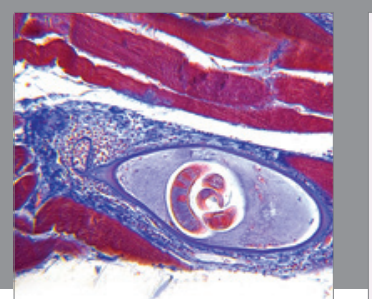

Gastroenterology Research and Practice

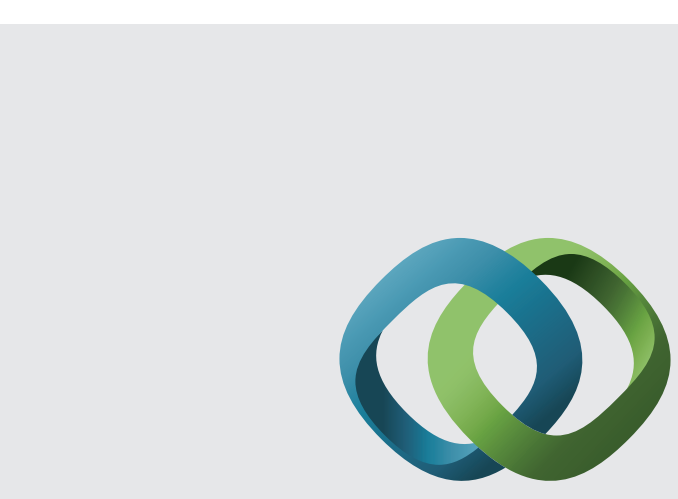

\section{Hindawi}

Submit your manuscripts at

http://www.hindawi.com
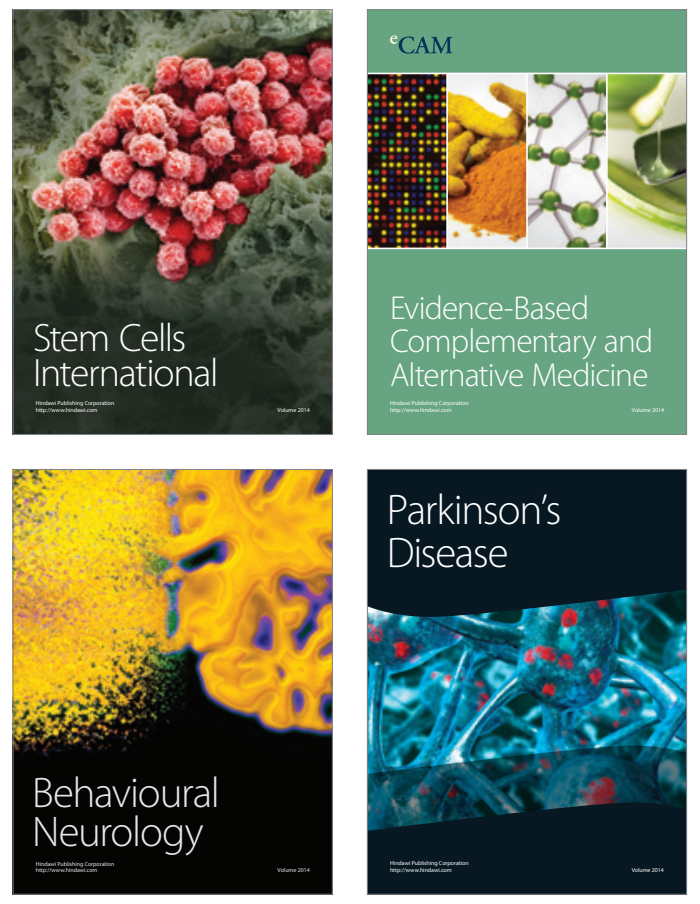
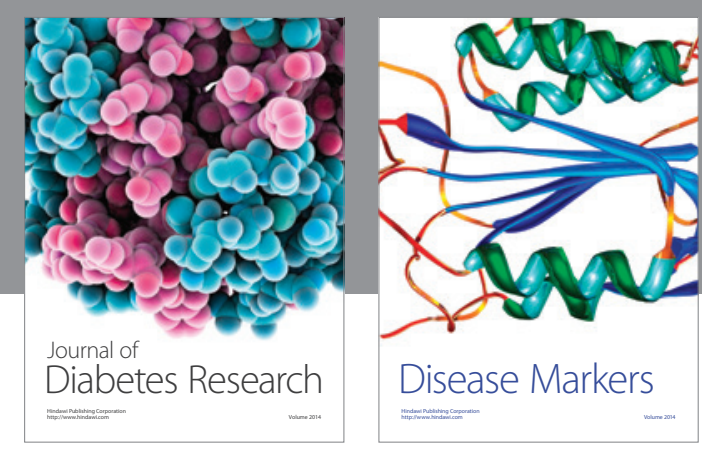

Disease Markers
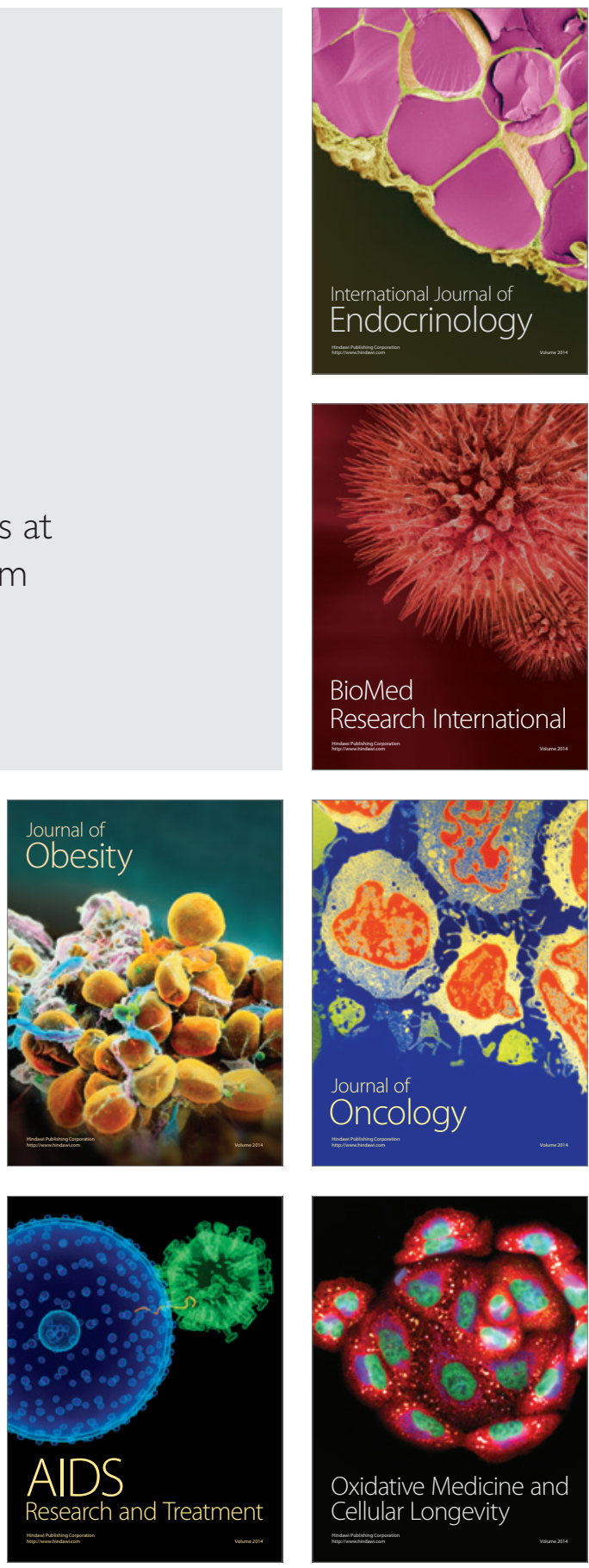